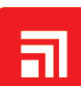 \\ Journal of Nuclear Physics, Material Sciences, Radiation and Applications

\section{Radiation Induced Reactions of Succinic Acid in Aqueous Solution: An Agent-Based Model}

\author{
Ana Leonor Rivera ${ }^{1,2 *}$, Sergio Ramos-Beltran ${ }^{1}$ and Alicia Negrón-Mendoza ${ }^{1}$ \\ ${ }^{1}$ Institute of Nuclear Sciences, National Autonomous University of Mexico, Coyoacan-04510, CDMX, Mexico \\ ${ }^{2}$ Center for Complexity Sciences, National Autonomous University of Mexico Ciudad Universitaria, Mexico City-04510, Mexico \\ *Email: ana.rivera@nucleares.unam.mx
}

\section{ARTICLE INFORMATION}

Received: October 9, 2019

Accepted: January 19, 2020

Published online: February 28, 2020

Keywords:

Radiation induced chemical reactions, Succinic Acid, Kinetics of reactions, Agent-based model

DOI: $10.15415 /$ jnp.2020.72014

\begin{abstract}
An approach to studying the formation of critical bio-organic compounds in the early Earth is to simulate in the laboratory possible processes that may occur in primitive scenarios. In this context, it can be studied the evolution of succinic acid in an aqueous media exposed to gamma radiation, as starting material produced more complex prebiotic molecules. To describe the products generated by the interaction of the different elements under radiation, there is a mathematical model that considers chemical reactions as nonlinear ordinary differential equations based on the mass balance of all the species, that has been implemented here by an agent-based model. In this simulation, each chemical species involved is considered as an agent that can interact with other species with known reaction rates, and the radiation is taken as a factor that promotes product formation. The results from the agentbased model are compared with the molar concentrations of succinic acid, and its products obtained in the lab. Simulation shows the exponential decomposition of succinic acid due to gamma radiation at room temperature in agreement with the laboratory model.
\end{abstract}

\section{Introduction}

Radiation-induced oxidation reactions play a fundamental role in the formation and decomposition of critical bioorganic molecules in the early Earth [1-3]. Even when early Earth conditions are difficult to simulate in the laboratory, there are few experimental setups studying the stability and formation of some compounds of biological importance under a high radiation field [4-6]. Succinic acid (HOOC- $\left.\left(\mathrm{CH}_{2}\right)_{2}-\mathrm{COOH}\right)$ is one of the molecules formed in prebiotic experiments [6], it has been found in carbonaceous chondrites $[7,8]$, it is essential in chemical evolution processes as a starting material for more complex molecules and participates in the metabolic pathways of living beings [9].

The goal of this work is to model the decomposition of succinic acid in an aqueous medium under a high radiation field. To describe the products generated by the interaction of the different species involved on succinic acid decomposition under radiation in an aqueous medium, we propose a mathematical model that describes the mass balance of all species involved considering chemical reactions as nonlinear ordinary differential equations (NODEs) $[5,10]$. This model is complicated due to the non-linear character of the equations that makes the model strongly dependent on initial conditions, the significant number of reactions involved, the coupling between equations, and by the fact that all the NODEs need to be solved simultaneously. To circumvent these issues, some authors have used MonteCarlo simulations [11], and in previous works, we have proposed an agent-based model [12-14]. In our model, the mass-balance equation includes sink terms (all the reactions leading to destruction) and source terms (all the reaction rates leading to production), and each chemical species involved is considered as an agent that can interact with other species with known reaction rates, radiation is taken as a factor that promotes a product's formation/destruction, and the temperature determines the diffusion speed of the agents.

\section{Experimental Setup}

The experimental setup is detailed described on previous works [15-17]. Succinic acid was crystallized twice from acetone and from distilled water, and it was dried in a vacuum oven at $60^{\circ} \mathrm{C}$ for 3 hours. Oxygen-free aqueous solutions of $0.1 \mathrm{~mol} \mathrm{dm}^{-3}$ succinic acid $(\mathrm{pH}=2.3)$ were prepared in modify glass syringes. The samples were irradiated at room 
temperature $(295 \mathrm{~K})$ in a ${ }^{60} \mathrm{Co}$-gamma source at different doses, from 0 to $500 \mathrm{kGy}$. Immediately after the irradiation, the non-volatile fraction was analyzed as methyl esters, according to [9]. Gas chromatography/mass spectrometry (CG-MS) was used to identify the reaction products, in particular succinic acid concentrations.

\section{Mathematical model}

Following the kinetics of the chemical reactions induced by the radiation, it is possible to monitor the concentration of the species. These chemical reactions can be modeled through differential equations that establish the mass balance for each species involved. The mass balance equations predict the expected change in the molar concentration of the species over time, and take into account all of the reaction rates leading to production (source terms) and to destruction (sink terms). Here we consider that each balance equation reflects the interaction of at most 2 species, and that such a reaction, whatever its rate, must lead to the production or consumption of a certain species, as proposed on a previous work [10]:

$$
\frac{d N_{i}(t)}{d t}=f_{i}+\sum_{j} \sum_{k} r_{j, k}^{(i)} N_{j}(t) N_{k}(t)-N_{i}(t) \sum_{m} r_{i, m}^{(i)} N_{m}(t),
$$

where $N_{i}$ denotes the molar concentration of the $i$-species at time $t, r_{j, k}^{(i)}$ is the reaction rate constant for the two-reactive species $j, k$, that produce the specie $i . f_{i}$, the radiolysis term, is an external source function of the radiation dose intensity $I_{d}$ and the bath-temperature $T$ :

$$
f_{\mathrm{i}}\left(I_{d}, T\right)=\frac{6.2 \times 10^{11}}{3.6 N_{A}} \frac{M_{i}}{M_{\mathrm{H}_{2} O}} G_{i} e^{-\frac{M_{i}}{2 N_{A} k T} I_{d}},
$$

where $M_{i}$ is the molecular mass of the specie, $M_{H_{2} O}$ is the molecular mass of water $(18.02 \mathrm{~g} / \mathrm{mol}), N_{A}$ is the Avogadro number $\left(6.022 \times 10^{23} \mathrm{~mol}\right), k$ is the Boltzmann constant $\left(1.38 \times 10^{-23} \mathrm{~m}^{2} \mathrm{kgs}^{-2} \mathrm{~K}^{-1}\right)$, and $G_{i}$ is the radiochemical constant that gives the number of species $i$ produced when $100 \mathrm{eV}$ are absorbed by the system $\left(0.47 \mu \mathrm{mol} \cdot \mathrm{J}^{-1}\right.$ for succinic acid $)$.

\section{Agent-based model}

The coupled system of Eqs. (1) can be solved using an agentbased model like the prey-predator one that we developed in previous works [12-14] where we generated a Python code to evaluate the molar concentrations of each species. In the code, each chemical species involved is considered as an agent that can interact with other species with known reaction rates, radiation is taken as a factor that promotes a product's formation/destruction, and the temperature determines the diffusion speed of the agents. The code initializes a region of $10,000^{*} 10,000$ pixels, with 100 randomly allocated points associated with succinic acid. Each position is checked during the next time instant, if two neighbor species can react in accordance to eqs. (1), the reaction takes place. The rate of reaction is determined by the $r_{j, k}^{(i)}$. After actualizing the entire net, the procedure is repeated until the steadystate is reached. In the program we can follow the molar concentration of each species as a function of time.

\section{Chemical radiation induced reaction on succinic acid}

The possible chemical reaction mechanism proposed in [17] is:
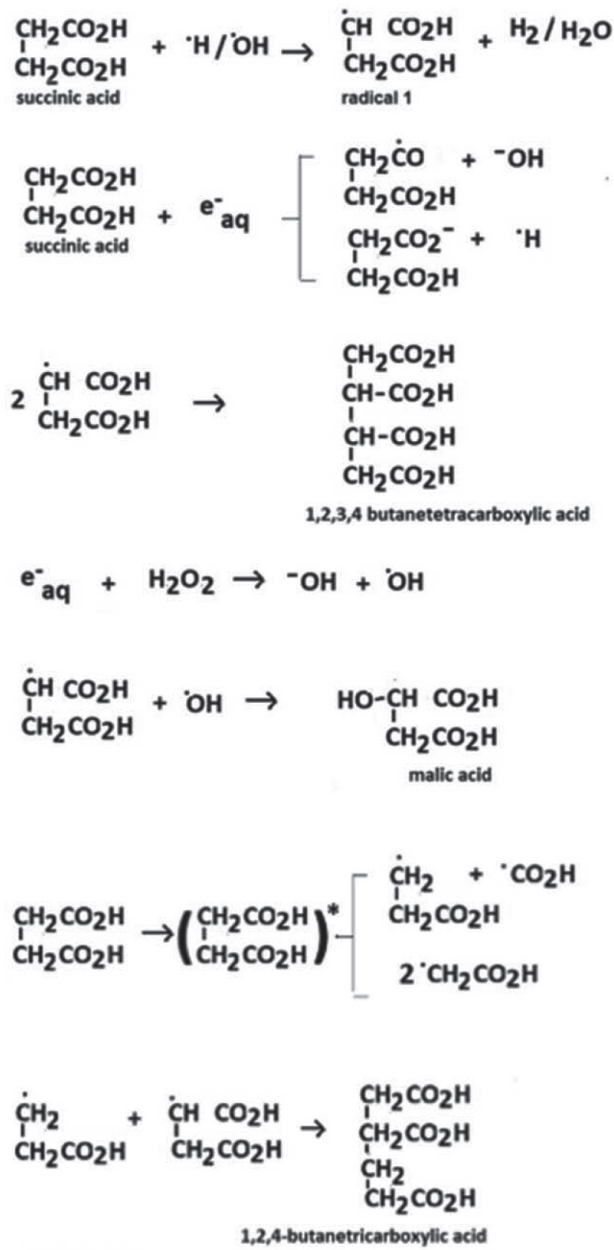


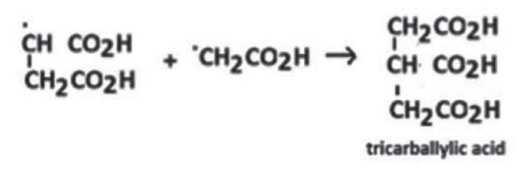

Reaction rates are known only for (2a), (2b) and (2d). We use our agent-based model to evaluate the unknown rates using experimentally determined concentration values. For this, we ran the model with different values of unknown rates until we reached the best experimental approximation (smallest mean square difference between experimental data and agent-based model).

\section{Results}

Our agent-based model is run for different gamma-radiation doses that induce the chemical decomposition reactions described in section 5 until the steady-state for succinic concentration is reached. Comparison of experimental data and the agent-based model for succinic acid concentration at different radiation doses is given in Fig. 1.

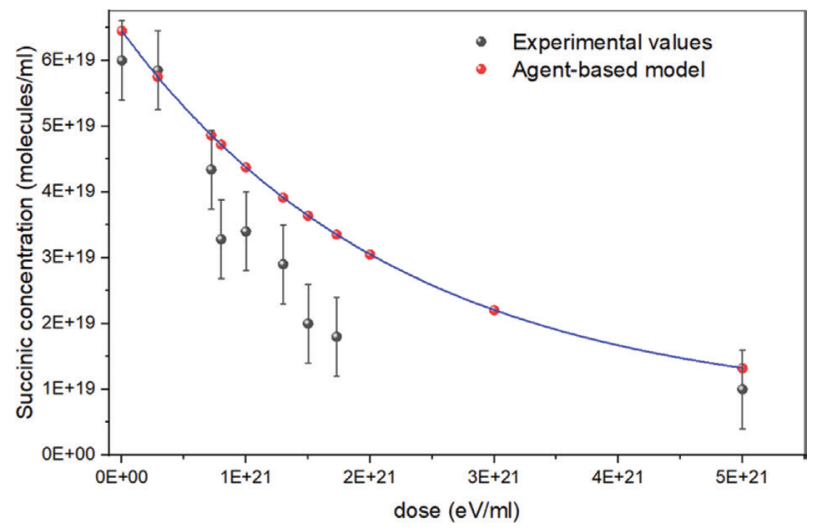

Figure 1: Succinic acid concentration as function of the gammaray dose intensity. Experimental results are in black while red points correspond to the steady-state value reached in the agentbased model. Blue line corresponds to the best exponential-decay fit of simulation points.

\section{Conclusion}

Succinic acid reactions induced by gamma radiation at room temperature were studied and compared with the concentrations determined by an agent-based model. This simple and robust model agrees with the experimental results for low and extreme radiation doses. In the intermediate region, simulation shows less succinic acid decomposition maybe due to bad approximation of the unknown reaction rates, especially for the reaction (2c) that is the most important.
Experimental and agent-base model approaches showed that succinic acid decomposed rapidly (exponential decay) in an environment with high gamma-radiation, thus, for the survival of this molecule and its further participation in the formation of more complex molecules, it is necessary a protection mechanism as the adsorption in clay minerals.

\section{Acknowledgements}

This work was partially funded by Universidad Nacional Autónoma de México through grants DGAPA PAPIIT IN113619, PAPIME PE103519, and by Consejo Nacional de Ciencia y Tecnología Fronteras FC-2016-1/2277. The funders had no role in study design, data collection and analysis, decision to publish, or preparation of the manuscript.

\section{References}

[1] A. Negrón-Mendoza and C. Ponnamperuma, Photochem. Photobiol. 36, 595 (1982). https://doi.org/10.1111/j.1751-1097.1982.tb04421.x

[2] A. Negrón-Mendoza, G. Albarran, S. Ramos, and E. Chacon, J. Biol. Phys. 20, 71 (1995). https://doi.org/10.1007/BF00700422

[3] M. Colín-García, A. Negrón-Mendoza, and S. RamosBernal, Astrobiol. 9, 279 (2009). https://doi.org/10.1089/ast.2006.0117

[4] S. Castillo, A. Negrón-Mendoza, Z. D. Draganic, and I. G. Draganic, Rad. Phys. Chem. 26, 437 (1985). https://doi.org/10.1016/0146-5724(85)90232-8

[5] J. Cruz-Castañeda, A. Negrón-Mendoza, D. Frías, M. Colín-García, et al., J. Radioanal. Nuc. Chem. 304, 219 (2015). https://doi.org/10.1007/s10967-014-3711-z

[6] S. L. Miller and H. C. Urey, Science 130, 245 (1959). https://doi.org/10.1126/science.130.3370.245

[7] G. U. Yuen and K. A. Kvenvolden, Nature 246, 301 (1973). https://doi.org/10.1038/246301a0

[8] J. G. Lawless, B. Zeiman, W. E. Pereira, R. E. Summons and A. M. Duffield, Nature 251, 40 (1974). https://doi.org/10.1038/251040a0

[9] A. Negrón-Mendoza and C. Ponnamperuma, Origins of Life 279, 191 (1976). https://doi.org/10.1007/BF00926937

[10] G. Sanchez-Mejorada, D. Frias, A. Negrón-Mendoza and S. Ramos-Bernal, Radiat. Meas. 43, 287 (2008). https://doi.org/10.1016/j.radmeas.2007.11.038

[11] V. P. Zhdanov, Surface Sci. Rep. 45, 231 (2002). https://doi.org/10.1016/S0167-5729(01)00023-1 
[12] A. L. Rivera, S. Ramos-Bernal and A. NegrónMendoza, J. Nucl. Phys. Mat. Sci. Rad. A. 6, 93 (2018). https://doi.org/10.15415/jnp.2018.61016

[13] A. L. Rivera, S. Ramos-Bernal, and A. NegrónMendoza, J. Nucl. Phys. Mat. Sci. Rad. A. 5, 15 (2017). https://doi.org/10.15415/jnp.2017.51002

[14] A. L. Rivera, S. Ramos-Bernal, and A. NegrónMendoza, J. Nucl. Phys. Mat. Sci. Rad. A. 4, 149 (2016). https://doi.org/10.15415/jnp.2016.41015

[15] I. G. Draganic and Z. D. Draganic, The radiation chemistry of water. (Academic Press, New York, pp 204-206, 1971).
[16] A. L. Meléndez-López, S. Ramos-Bernal, and M. L. Ramírez-Vázquez, AIP Conf. Proc. 1607, 111 (2014). https://doi.org/10.1063/1.4890710

[17] A. Negrón-Mendoza, M. Colín-García, and S. RamosBernal, J. Radioanal. Nuc. Chem. 318, 2279 (2018). https://doi.org/10.1007/s10967-018-6197-2 


\section{Journal of Nuclear Physics, Material Sciences, Radiation and} Applications

Chitkara University, Saraswati Kendra, SCO 160-161, Sector 9-C, Chandigarh, 160009, India

Volume 7, Issue 2

February 2020

ISSN 2321-8649

Copyright: [C 2020 Ana Leonor Rivera et al.] This is an Open Access article published in Journal of Nuclear Physics, Material Sciences, Radiation and Applications (J. Nucl. Phy. Mat. Sci. Rad. A.) by Chitkara University Publications. It is published with a Creative Commons Attribution- CC-BY 4.0 International License. This license permits unrestricted use, distribution, and reproduction in any medium, provided the original author and source are credited. 